\title{
Mathematical Efficiency Modeling of Static Power Converters
}

\author{
Fabrício Hoff Dupont \\ Regional Integrated University \\ of Upper Uruguai and Missions (URI) \\ Av. Assis Brasil, 709, 98400-000 \\ Frederico Westphalen, RS, Brazil \\ Contact: fhd@ieee.org
}

\author{
Jordi Zaragoza Bertomeu \\ Technical University of Catalonia (UPC) \\ C. Colom, 1, 08222, \\ Terrassa, Barcelona, Spain
}

\author{
Cassiano Rech \\ and José Renes Pinheiro \\ Federal University of Santa Maria (UFSM) \\ Av. Roraima, 1000, 97105-900 \\ Santa Maria, RS, Brazil
}

\begin{abstract}
This paper presents a review and a comparative analysis between mathematical models for the efficiency of power converters. Two different types of models are considered, being one for converters subject solely for output power variations, and a second one also considering input voltage variations. Both cases are particularly important for systems fed by renewable sources as photovoltaic panels or wind turbines. Knowledge of the appropriate models is of interest in the development of high-performance systems, allowing one to optimize the global efficiency of the converter system. Experimental results concludes the comparison between the investigated models and a discussion of pros and cons of each model are presented, providing to authors a better choice for modeling the efficiency behavior of power converters.
\end{abstract}

\section{INTRODUCTION}

It is known that the efficiency of a system is defined by the ratio between the power delivered to the loads (output power $p_{\text {out }}$ ), and the power absorbed from the source (input power $\left.p_{\text {in }}\right)$. The losses in the conversion process are manifested in several ways, either by switching or conduction of the semiconductors, ancillary systems such as drive, protection or signaling circuits, among others [1], [2].

The total losses composition of a converter varies, besides its technological and constructive characteristics, in function of its operating points. For low power levels, losses in the driving circuits of the switches may be more significant and also approximately constant. As power level increases, losses related to switching and conduction of semiconductors, and to magnetic devices, starts to become more significant.

Development of mathematical models is a task that commonly faces the need of a balance between complexity and precision. For most physical systems, higher the required accuracy, greater the complexity of its mathematical model. The "incompatibility principle" states that with increasing complexity, our capacity to do accurate descriptions of a system behavior decrease until a threshold beyond which simplicity and accuracy become mutually exclusive features [3]. Thus, it can be said that a good mathematical model is the one that allows sufficient simplicity without excessively sacrificing accuracy. For the task of modeling the efficiency of power converters as a function of the input voltage or the power processed one may use different approaches. In this paper the modeling will be evaluated by means of a set of samples which may be obtained either experimentally or algebraic, from an in-depth analysis of the power conversion losses.

For optimized and high performance systems, knowledge of an appropriate efficiency model for power converters is one of the first steps to apply the optimization methodologies. In [4] the efficiency curve is employed to identify better regions of operation for systems of parallel converters such that the overall efficiency of the system is improved. Also, in [5] mathematical models for efficiency of power converters are employed in an algorithm to obtain the optimal power sharing so that systems of parallel converters are able to operate with maximum efficiency throughout its entire load range. Both cases employ the converter efficiency models as a key tool for improving the performance of energy conversion systems.

The aim of this paper is to review, evaluate and compare different approaches for modeling of power converters efficiency. Initially, the analysis of models that consider only variations in the processed output power is presented, the most common case and which is called here as unidimensional models. In the sequence, the analysis is extended for models that consider variations in two dimensions. More specifically, both input voltage and processed power variations are taken into account. This is frequently a recurring situation in systems powered by photovoltaic modules, and called here as bidimensional models. After, the investigated models are compared by means of experimental results obtained with a prototype of a boost converter with a rated output power of $250 \mathrm{~W}$ and an output voltage of $325 \mathrm{~V}$.

\section{UNIDIMENSIONAL MODELS}

A common steady-state analysis of converters involves tracing of an efficiency curve as a function of the processed power, going from a minimum value to the rated power of the converter. Thus, efficiency or losses can be evaluated for different operating points.

In this sense, it is verified that the most relevant variable for the efficiency modeling of power converters is the processed power, and it is known that in function of the different operating points the efficiency can suffer considerable 
variations. Depending on the application characteristics in which this converter is inserted, power may be the unique parameter that suffers variations. So, the first efficiency models investigated in this paper are for these unidimensional cases.

One of the simplest approaches is presented by Keating et al. [6], which proposes a linear interpolation between efficiency samples. These samples can be obtained both experimentally and by algebraic methods, modeling and analyzing theoretic losses for each part of the converter. The interpolation proposed by authors is defined by

$$
\eta(p)=\eta_{\text {low }}+\frac{\left(p-p_{\text {low }}\right)\left(\eta_{\text {upp }}-\eta_{\text {low }}\right)}{\left(p_{\text {upp }}-p_{\text {low }}\right)}
$$

being $p$ the power for which the efficiency will be evaluated, $p_{\text {low }}$ and $p_{\text {upp }}$ the power for lower and upper samples, as well as $\eta_{\text {low }}$ and $\eta_{\text {upp }}$ the efficiency values for the lower and upper points, respectively. However, this approach requires the availability of a table of efficiency samples and its precision may be seriously compromised if the efficiency curve is inappropriate sampled.

If the goal of efficiency modeling is to obtain a single mathematical expression that depends solely of numerical coefficients that may be obtained by curve fitting, a negative point of this previous approach is the discontinuities on derivatives and the need to store all samples in a table. Those features make it difficult, or even impossible, to apply interpolation models in many numerical analysis methods.

Driesse et al. [7] presents a continuous and simple model, where the efficiency of power converters is approximated by the second order function

$$
\eta(p)=\alpha_{0}+\alpha_{1} p+\alpha_{2} p^{2}
$$

being $\alpha_{0}, \alpha_{1}$ and $\alpha_{2}$ coefficients to be obtained by curve fitting. Although presenting good correlation of the fitted curve for upper half and around the rated power, one may observe serious limitations to represent the lower part of the efficiency curve. This behavior may be unacceptable for some applications.

Such problems do not occur in the model proposed by Jantsch et al. [8], where the authors approximate the efficiency curve by means of the function

$$
\eta(p)=\frac{p}{p+\left(k_{0}+k_{1} p+k_{2} p^{2}\right)}
$$

being $k_{0}, k_{1}$ and $k_{2}$ the coefficients to be fitted. Comparing to the previous, this model has the same number of coefficients, but naturally ensures zero efficiency at the origin of power axis. This feature significantly improves the correlation between samples and the fitted curve.

Another model is proposed by Dupont et al. [5], in which the efficiency curve is treated as the rational function

$$
\eta(p)=\frac{\alpha_{1} p+\alpha_{0}}{p^{2}+\beta_{1} p+\beta_{0}}
$$

being $\alpha_{1}, \alpha_{0}, \beta_{1}$, and $\beta_{0}$ the coefficients to be adjusted. Although with more coefficients, this model features good fitting capabilities throughout the entire power range, however may show some representation problems for efficiency near the origin. This problems is due to the self characteristic of the equation, but can be bypassed with the addition of a zero efficiency sample. This sample can represent, for example, the minimum working power of the converter, below it the converter is turned off.

\section{BIDIMENSIONAL MODELS}

Many applications, especially for systems fed by renewable sources, it is not the power processed that only suffers variations. In these cases the input voltage may present values between a range that cannot not be neglected, and directly interfere the efficiency of converters. For those applications the unidimensional models are not sufficient, and bidimensional ones are necessary.

For such applications Chivelet et al. [9] proposes a model where the efficiency is determined by an equivalent electrical circuit. A series resistance $R_{s}$ and a parallel resistance $R_{p}$ are included between source and load to represent ohmic losses and self consumption of the converter, respectively. This model is described by

$$
\eta\left(p, v_{\text {in }}\right)=\frac{2 p R_{s}}{v_{\text {in }}^{2}} \frac{1}{1-\sqrt{1-4 \frac{R_{s}}{v_{\text {in }}^{2}}\left(p+\frac{v_{\text {out }}^{2}}{R_{p}}\right)}}
$$

where $R_{s}$ and $R_{p}$ are the coefficients to be obtained by a surface fitting algorithm

Another approach is presented by Rampinelli et al. [10], that modifies coefficients $k_{0}, k_{1}$ and $k_{2}$ of (3), making them functions of the input voltage. The proposed model is defined by

$$
\eta\left(p, v_{\text {in }}\right)=\frac{p}{p+\left(k_{0}\left(v_{\text {in }}\right)+k_{1}\left(v_{\text {in }}\right) p+k_{2}\left(v_{\text {in }}\right) p^{2}\right)}
$$

being $k_{0}\left(v_{\text {in }}\right), k_{1}\left(v_{\text {in }}\right)$ and $k_{2}\left(v_{\text {in }}\right)$ functions that describes the behavior $k_{0}, k_{1}$ e $k_{2}$ for input voltage variations. However, these functions are not specified. Assuming a linear dependency of efficiency with the input voltage, one may represent this behavior defining the functions

$$
\begin{aligned}
& k_{0}^{\prime}\left(v_{\text {in }}\right) \triangleq k_{0,0}+k_{0,1} v_{\text {in }} \\
& k_{1}^{\prime}\left(v_{\text {in }}\right) \triangleq k_{1,0}+k_{1,1} v_{\text {in }} \\
& k_{2}^{\prime}\left(v_{\text {in }}\right) \triangleq k_{2,0}+k_{2,1} v_{\text {in }}
\end{aligned}
$$

being $k_{0,0}, k_{0,1}, k_{1,0}, k_{1,1}, k_{2,0}$ and $k_{2,1}$ the coefficients to be determined by a numeric algorithm for surface fitting. Thus, the efficiency of the converters may be modeled by the surface

$$
\eta\left(p, v_{\text {in }}\right)=\frac{p}{p+\left(k_{0}^{\prime}\left(v_{\text {in }}\right)+k_{1}^{\prime}\left(v_{\text {in }}\right) p+k_{2}^{\prime}\left(v_{\text {in }}\right) p^{2}\right)}
$$

named hereinafter as linear bidimensional model, due to the model dependency with the input voltage.

For converters in which input voltage cannot be linearly represented, one can opt by a model whose coefficients varies 
in a quadratic way both with the input voltage and with output power. For this, the following functions are defined

$$
\begin{aligned}
& k_{0}^{\prime \prime}\left(v_{\text {in }}\right) \triangleq k_{0,0}+k_{0,1} v_{\text {in }}+k_{0,2} v_{\text {in }}^{2} \\
& k_{1}^{\prime \prime}\left(v_{\text {in }}\right) \triangleq k_{1,0}+k_{1,1} v_{\text {in }}+k_{1,2} v_{\text {in }}^{2} \\
& k_{2}^{\prime \prime}\left(v_{\text {in }}\right) \triangleq k_{2,0}+k_{2,1} v_{\text {in }}+k_{2,2} v_{\text {in }}^{2}
\end{aligned}
$$

to apply at (6) and results in the double quadratic bidimensional model in which $k_{0,0 \ldots 2}, k_{1,0 \ldots 2}$ and $k_{2,0 \ldots 2}$ are the coefficients to be determined. The adjusted surface is then modeled by

$$
\eta\left(p, v_{\text {in }}\right)=\frac{p}{p+\left(k_{0}^{\prime \prime}\left(v_{\text {in }}\right)+k_{1}^{\prime \prime}\left(v_{\text {in }}\right) p+k_{2}^{\prime \prime}\left(v_{\text {in }}\right) p^{2}\right)}
$$

It is worth to notice that although this model allows to improve the approximation of experimental samples to the fitted surface, there is a high number of coefficients to be determined. As a consequence, a larger number of samples should be passed to the fitting algorithm.

Finally, Driesse et al. [7] proposes an improved model, as named by authors, in which the quadratic term, that do not have a physical meaning, is substituted by the inverse of input voltage to represent the existence of multiple dependencies with this variable. The model proposed by authors is defined by

$$
\eta\left(p, v_{\text {in }}\right)=\frac{p}{p+b_{0}\left(v_{\text {in }}\right)+b_{1}\left(v_{\text {in }}\right) p+b_{2}\left(v_{\text {in }}\right) p^{2}}
$$

where the functions $b_{0}\left(v_{\text {in }}\right), b_{1}\left(v_{\text {in }}\right)$ and $b_{2}\left(v_{\text {in }}\right)$ are

$$
\begin{aligned}
& b_{0}\left(v_{\text {in }}\right)=b_{0,0}+b_{0,1}\left(v_{\text {in }}-1\right)+b_{0,2}\left(\frac{1}{v_{\text {in }}}-1\right) \\
& b_{2}\left(v_{\text {in }}\right)=b_{2,0}+b_{1,1}\left(v_{\text {in }}-1\right)+b_{1,2}\left(\frac{1}{v_{\text {in }}}-1\right) \\
& b_{2}\left(v_{\text {in }}\right)=b_{3,0}+b_{2,1}\left(v_{\text {in }}-1\right)+b_{2,2}\left(\frac{1}{v_{\text {in }}}-1\right)
\end{aligned}
$$

being $b_{0,0 \ldots 2}, b_{1,0 \ldots 2}, b_{2,0 \ldots 2}$ the coefficients to be fitted.

\section{EXPERIMENTAL RESUlTS}

Aiming to evaluate the efficiency models for curves of surfaces investigated in this paper, a comparative analysis is presented in this section. For this, experimental tests have been conducted to obtain a set of experimental efficiency samples for a $10 \mathrm{kHz}$ boost converter with a rated output voltage of $325 \mathrm{~V}$ at $250 \mathrm{~W}$. The prototype is depicted by Figure 1, and a full table of specifications is given by Table I.

Efficiency samples has been acquired with a Yokogawa WT1600 power analyzer after the converter reaches a thermal steady state. Input voltage has been varied in the range of $110 \mathrm{~V}$ to $250 \mathrm{~V}$, with a $20 \mathrm{~V}$ step. On the other hand, the load analyzed was of $30 \mathrm{~W}, 50 \mathrm{~W}, 70 \mathrm{~W}, 102 \mathrm{~W}$, $146 \mathrm{~W}, 222 \mathrm{~W}, 232 \mathrm{~W}$, and $282 \mathrm{~W}$, which corresponds to $12 \%, 20 \%, 28 \%, 41 \%, 59 \%, 89 \%, 93 \%$, and $113 \%$ of the converter rated power, respectively. Thus, a total of 64 efficiency samples has been acquired.

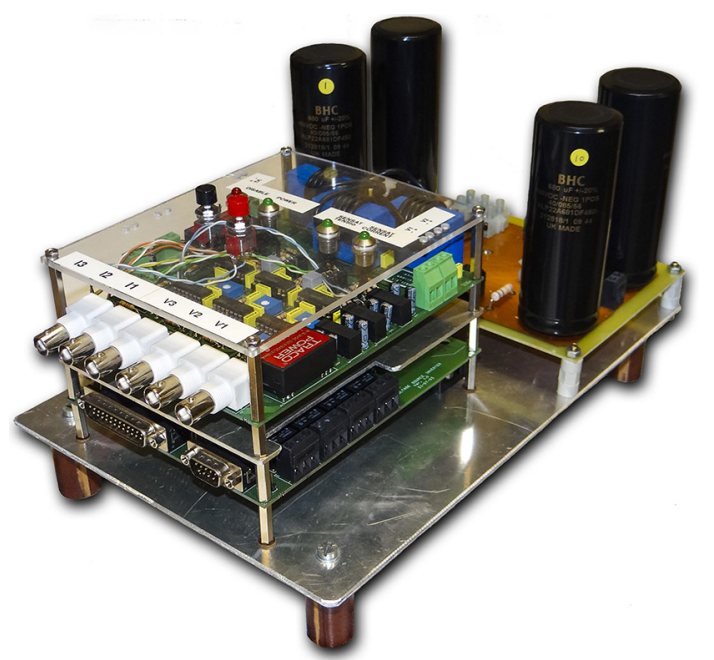

Figure 1. Prototype of the boost converter employed for the experimental analysis of the efficiency models.

Table I

MAIN SPECIFICATIONS OF THE PROTOTYPE.

\begin{tabular}{lc}
\hline Parameter & Value \\
\hline Input voltage & $110 \mathrm{~V}$ to $250 \mathrm{~V}$ \\
Rated input voltage & $V_{\text {in, nom }}=190 \mathrm{~V}$ \\
Rated output voltage & $V_{\text {out,nom }}=325 \mathrm{~V}$ \\
Rated duty cycle & $D_{\text {nom }}=0.415$ \\
Duty cycle range & $0.23 \leq D \leq 0.66$ \\
Inductor & $L=6 \mathrm{mH}$ \\
Output capacitance & $C=680 \mu \mathrm{F}$ \\
Switching frequency & $F_{s}=10 \mathrm{kHz}$ \\
Rated output power & $P_{\text {conv,nom }}=250 \mathrm{~W}$ \\
Power module & Mitsubishi PM15CZF120 \\
\hline
\end{tabular}

Table II

EXPERIMENTAL EFFICIENCY SAMPLES FOR THE ANALYZED PROTOTYPE.

\begin{tabular}{ccccccccc}
\hline & \multicolumn{7}{c}{ Output power (\%) } \\
\cline { 2 - 9 }$V_{\text {in }}$ & 12 & 20 & 28 & 41 & 59 & 89 & 93 & 113 \\
\hline 110 & 72.72 & 79.22 & 83.39 & 87.59 & 90.47 & 92.67 & 92.84 & 93.55 \\
130 & 74.35 & 79.93 & 83.28 & 87.51 & 90.55 & 92.92 & 93.12 & 93.87 \\
150 & 76.76 & 81.47 & 84.09 & 87.64 & 90.79 & 93.20 & 93.39 & 91.18 \\
170 & 79.11 & 83.03 & 85.36 & 88.08 & 91.21 & 93.59 & 93.80 & 94.56 \\
190 & 81.77 & 85.09 & 86.87 & 89.14 & 91.85 & 94.06 & 94.31 & 95.03 \\
210 & 84.16 & 87.35 & 88.57 & 90.37 & 92.78 & 94.81 & 94.97 & 95.62 \\
230 & 87.84 & 89.83 & 90.29 & 91.94 & 93.93 & 95.68 & 95.83 & 96.33 \\
250 & 90.24 & 93.11 & 91.41 & 93.77 & 95.42 & 96.68 & 96.83 & 97.21 \\
\hline
\end{tabular}

Tests have been conducted in an increasing order for power, and on a decreasing order for input voltage. For each load configuration an input voltage sweep has been carried out. In other words, for each load value, the efficiency was sampled for all input voltage values. In this way the thermal equilibrium has been achieved more quickly. Obtained results are presented by Table II.

The numerical adjust for the samples to the curves or surfaces of the efficiency models has been carried out with the aid of a computational tool employing the numerical algorithm of Levenberg-Marquardt [11]. Following sections 


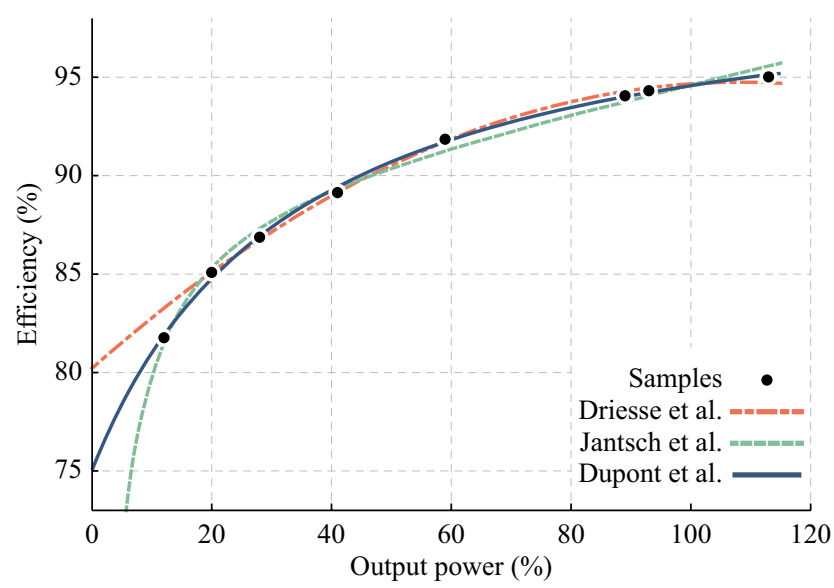

Figure 2. Comparison between fitted curves of the unidimensional models for the efficiency samples of the boost converter from Table I.

present a comparative analysis between the unidimensional and bidimensional models investigated in this paper.

\section{A. Comparison of Unidimensional Models}

To compare the unidimensional models, all the efficiency samples that comprises the entire load range of the nominal input voltage has been considered. Applying the curve fitting algorithm to the model of Driesse et al. (2), the obtained coefficients are

$$
\left\{\begin{array}{l}
\alpha_{0}=802.1620 \times 10^{-3} \\
\alpha_{1}=268.4868 \times 10^{-3} \\
\alpha_{2}=-123.9831 \times 10^{-3}
\end{array}\right.
$$

while the coefficients obtained after fitting the model of Jantsch et al. (3) to the experimental efficiency samples are

$$
\left\{\begin{array}{l}
k_{0}=14.8371 \times 10^{-3} \\
k_{1}=111.7171 \times 10^{-3} \\
k_{2}=-69.4710 \times 10^{-3}
\end{array}\right.
$$

Finally, the curve fitting for the model of Dupont et al. (4) has resulted in the coefficients

$$
\left\{\begin{array}{l}
\alpha_{0}=48.8087 \\
\alpha_{1}=188.9739 \\
\beta_{0}=65.0315 \\
\beta_{1}=185.3915
\end{array}\right.
$$

A comparison between fitted curves is depicted by Figure 2, which also shows the sample values that have been employed by the fitting process. From these results, one can verify that the root-mean-square error $\left(\mathrm{RMS}_{\mathrm{e}}\right)$ between samples and the fitted curve for the model of Driesse et al. is $3.904 \times 10^{-3}$, while for the model of Jantsch et al. is $4.827 \times 10^{-3}$. Finally, the $\mathrm{RMS}_{\mathrm{e}}$ for the model of Dupont et al. is $2.409 \times 10^{-3}$.
Two aspects must be highlighted from the analysis of the results. First, with regard the smallest error between samples and fitted curves, one can verify that the model of Dupont et al. is the one that features the smallest value of $\mathrm{RMS}_{\mathrm{e}}$, aided by a greater number of coefficients. Besides this, the model of Jantsch et al. also features good correlation to the samples, as observed in the curves of Figure 2. Both models have good representation for most curves, being the model of Jantsch et al. the one with easier convergence once it naturally crosses by zero and has one coefficient less. On the other hand, results obtained with the model of Dupont et al. may be improved if a zero efficiency sample is added to the set. This can be the case, for example, to consider the inclusion of a minimal power level, which below this value the converter is turned off. This kind of situation cannot be adequately represented by the model of Jantsch et al.

Among the analyzed models, the model of Driesse et al. is the one that features the poorest results, mainly for low power. In the case of this model, if a zero efficiency is added, the fitting curve algorithm has problems to converge below an acceptable error, once the model itself is unable to represent a zero efficiency if $\alpha_{0} \neq 0$. Thus, the model of Driesse et al. is recommended to only represent converters whose main application deals with loads with low variations and near the rated power. It is not interesting, for example, to model the efficiency of converters applied to renewable sources, which often operate at low power and have significant power variations.

\section{B. Comparison of Bidimensional Models}

To compare the bidimensional models investigated in this paper the whole set of experimental efficiency samples presented by Table II has been considered. Adjusting these samples to the model of Chivelet et al., defined by (5) the following coefficients have been obtained

$$
\left\{\begin{array}{l}
R_{s}=1.0025 \times 10^{-3} \\
R_{p}=19.9771 \times 10^{3}
\end{array}\right.
$$

Adjusting the surface described by the linear bidimensional model, defined by (6), to the efficiency samples results in the coefficients

$$
\left\{\begin{array}{l}
k_{0,0}=63.4175 \times 10^{-3} \\
k_{0,1}=-46.3601 \times 10^{-3} \\
k_{1,0}=76.7418 \times 10^{-3} \\
k_{1,1}=14.1165 \times 10^{-3} \\
k_{2,0}=-30.5100 \times 10^{-3} \\
k_{2,1}=-23.0776 \times 10^{-3}
\end{array}\right.
$$

while the coefficients obtained for the double quadratic 


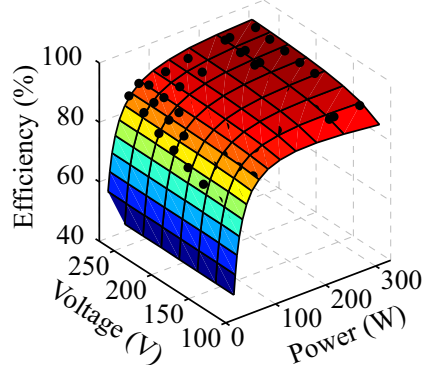

(a)

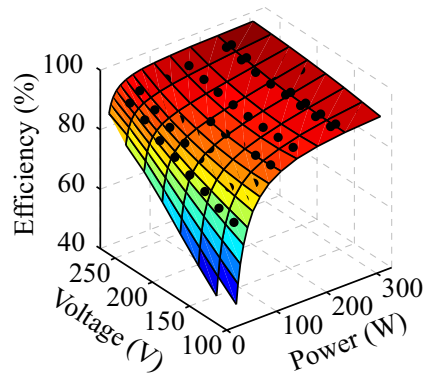

(c)

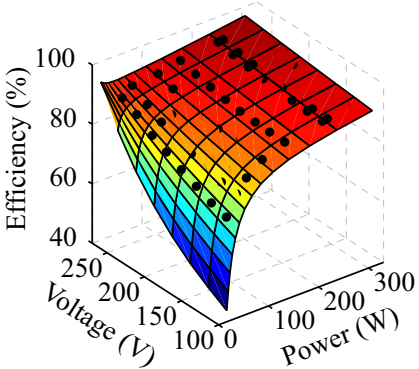

(b)

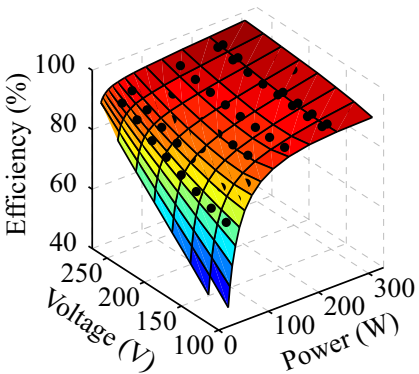

(d)

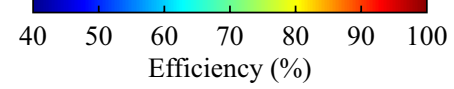

Figure 3. Experimental samples and fitted efficiency surfaces for the investigated bidimensional models.

bidimensional model, defined (14), are

$$
\left\{\begin{array}{l}
k_{0,0}=112.9645 \times 10^{-3} \\
k_{0,1}=-159.9778 \times 10^{-3} \\
k_{0,2}=60.2896 \times 10^{-3} \\
k_{1,0}=-360.6354 \times 10^{-3} \\
k_{1,1}=1.0194 \\
k_{1,2}=-534.7211 \times 10^{-3} \\
k_{2,0}=325.6128 \times 10^{-3} \\
k_{2,1}=-847.7486 \times 10^{-3} \\
k_{2,2}=438.1434 \times 10^{-3}
\end{array}\right.
$$

Finally, the efficiency surface fitting for the improved model of Driesse et al. (15) has resulted in the coefficients

$$
\left\{\begin{array}{l}
b_{0,0}=13.2619 \times 10^{-3} \\
b_{0,1}=3.7718 \times 10^{-3} \\
b_{0,2}=40.0147 \times 10^{-3} \\
b_{1,0}=124.3920 \times 10^{-3} \\
b_{1,1}=-428.5556 \times 10^{-3} \\
b_{1,2}=-351.3761 \times 10^{-3} \\
b_{2,0}=-85.3099 \times 10^{-3} \\
b_{2,1}=343.4734 \times 10^{-3} \\
b_{2,2}=292.4794 \times 10^{-3}
\end{array}\right.
$$

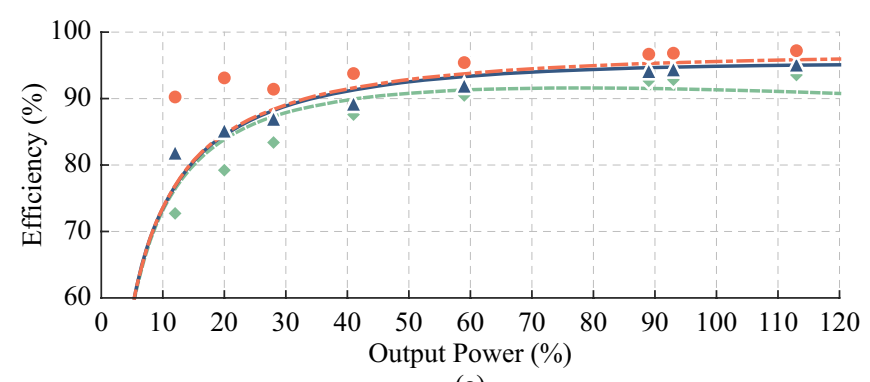

(a)

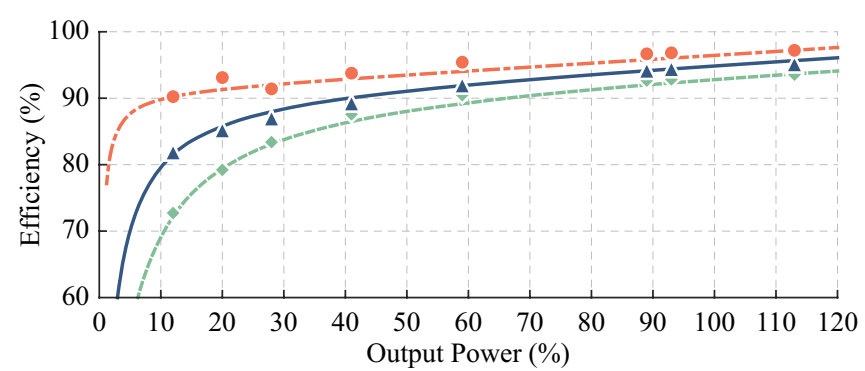

(b)

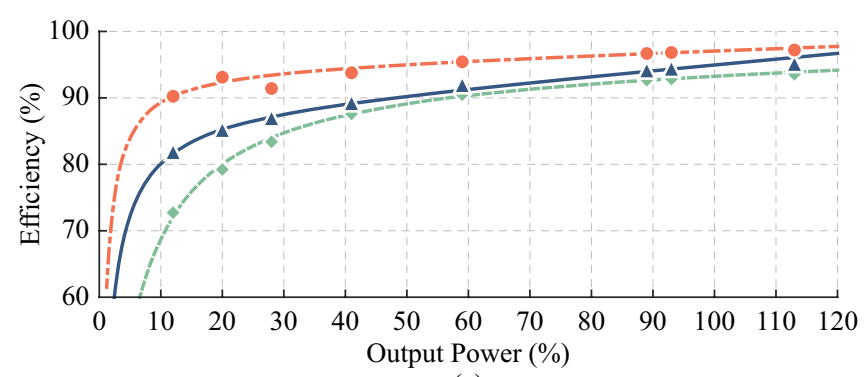

(c)

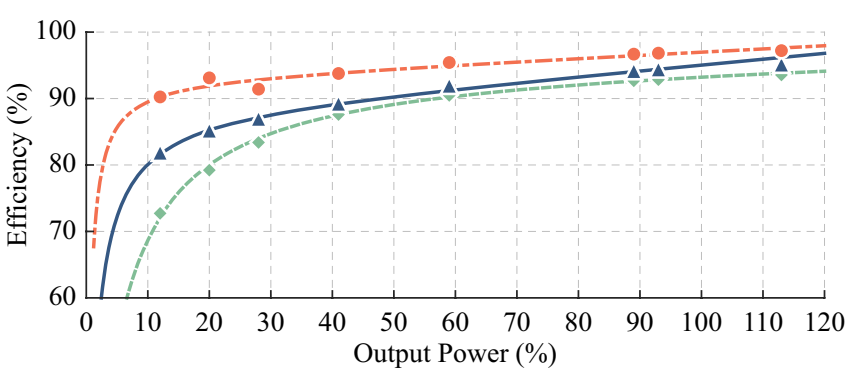

(d)

$$
\begin{aligned}
& \text { Modeled curve: ----- } v_{\min }-v_{\text {nom }}---v_{\max } \\
& \text { Experimental samples: } \quad v_{\min } \Delta v_{\text {nom }} \bullet v_{\max }
\end{aligned}
$$

Figure 4. Comparative detail between samples and the fitted surface on efficiency models for the input voltages of $110 \mathrm{~V}\left(v_{\min }\right), 190 \mathrm{~V}\left(v_{\text {nom }}\right)$ and $250 \mathrm{~V}\left(v_{\max }\right)$ (a) Chivelet et al.; (b) linear bidimensional; (c) double quadratic; (d) Driesse et al..

Figure 3 depicts the comparison between experimental samples and the fitted surfaces by the bidimensional models presented. A different point of view is also shown by Figure 4, which compares the samples with the efficiency curves for the minimum $\left(v_{\min }\right)$, nominal $\left(v_{\text {nom }}\right)$, and maximum $\left(v_{\max }\right)$ input voltages. From the obtained results, one can determine that for the model of Chivelet et al. the value of $\mathrm{RMS}_{\mathrm{e}}$ 
is $57.1668 \times 10^{-3}$. For the linear bidimensional model the $\mathrm{RMS}_{\mathrm{e}}$ is $8.3286 \times 10^{-3}$, while the $\mathrm{RMS}_{\mathrm{e}}$ for the double quadratic model is $5.1435 \times 10^{-3}$. Finally, the fitting by the improved model of Driesse et al. for the efficiency samples resulted in a $\mathrm{RMS}_{\mathrm{e}}$ of $5.0930 \times 10^{-3}$.

From the experimental samples and the presented analysis one can verify that the equivalent circuit model of Chivelet et al. presents the highest approximation error. On the other hand, the other three models provide a good correlation between efficiency samples and the fitted surface. Although if the efficiency surface feature a non-linear behavior, the linear bidimensional model may result in unacceptable approximation errors, while the double quadratic and the improved models may still provide good correlation. This improvement in precision has the cost of additional samples to fit the additional coefficients, and an small increase in computational time. But those cons are negligible in comparison with the major accuracy improvement.

\section{CONCLUSION}

This paper presented a review and a comparative analysis between different mathematical models for efficiency modeling of power converters. Main advantages and disadvantages of each model, as well as the error between samples and the fitted efficiency curve, or surface, has been pointed out based on a series of experimental results. For unidimensional cases the results pointed that both models of Jantsch et al. and Dupont et al. have good correlation between efficiency samples and the fitted curve. The former naturally crosses by zero for no power, while the second one is able to consider a specific power level where below it the converter turns itself off. For bidimensional cases, one could verify that the models with better correlation with the experimental samples are the double quadratic and the model of Driesse et al. In this case, both models are able to fit surfaces with nonlinear dependencies with the input voltage. Finally, it is worth to mention that even causing major impact over the converter efficiency, power and the input voltage are not the only variables associated with losses. The converter operating temperature also produces variations on the efficiency, adding a third variable to the problem and increasing the complexity of the model. In this sense, extensions of the presented analysis for tridimensional models are under investigation.

\section{ACKNOWLEDGMENT}

This work is supported by CNPq (National Council of Scientific and Technological Development), CAPES (Brazilian Commission for Higher Education), FAPERGS (Foundation for Supporting Research from the State of Rio Grande do Sul), and RURALGRID project on CTP frame with support of the Comissionant per a Universitats i Recerca del Departament d'Innovació, Universitats i Empresa of the Generalitat de Catalunya.

\section{REFERENCES}

[1] K. Raggl, T. Nussbaumer, G. Doerig, J. Biela, and J. W. Kolar, "Comprehensive design and optimization of a high-power-density singlephase boost PFC," IEEE Transactions on Industrial Electronics, vol. 56 no. 7, pp. 2574-2587, 2009.

[2] J. Biela, U. Badstuebner, and J. W. Kolar, "Design of a 5-kW, 1-U, $10-\mathrm{kW} / \mathrm{dm} 3$ resonant DC-DC converter for telecom applications," IEEE Transactions on Power Electronics, vol. 24, no. 7, pp. 1701-1710, 2009.

[3] M. N. Cirstea, A. Dinu, J. G. Khor, and M. McCormick, Neural and Fuzzy Logic Control of Drive and Power Systems. Oxford: Newnes, 2002.

[4] S. Effler, M. Halton, and K. Rinne, "Efficiency-based current distribution scheme for scalable digital power converters," IEEE Transactions on Power Electronics, vol. 26, no. 4, pp. 1261-1269, 2011.

[5] F. H. Dupont, J. Zaragoza, C. Rech, and J. R. Pinheiro, "A new method to improve the total efficiency of parallel converters," in Brazilian Power Electronics Conference (COBEP), 2013, pp. 210-215.

[6] L. Keating, D. Mayer, S. McCarthy, and G. T. Wrixon, "Concerted action on computer modeling and simulation," in European Photovoltaic Solar Energy Conference, Lisboa, Portugal, 1991.

[7] A. Driesse, P. Jain, and S. Harrison, "Beyond the curves: modeling the electrical efficiency of photovoltaic inverters," in 33rd IEEE Photovoltaic Specialists Conference (PVSC '08), San Diego, CA, 2008, pp. 1-6.

[8] M. Jantsch, H. Schimidt, and J. Schmid, "Results of the Concerted Action on Power Conditioning and Control," in 11th European Photovoltaic Solar Energy Conference, Montreux, Switzerland, 1992, pp. 1589-1593.

[9] N. M. Chivelet, F. Chenlo-Romero, and M. C. Alonso-Garcia, "Modelado y fiabilidad de los inversores para instalaciones fotovoltaicas autónomas a partir de medidas con cargas resistivas y reactivas," in 7 th Congresso Ibérico de Energía Solar, España, 1994, pp. 463-468.

[10] G. A. Rampinelli, "Estudo de características elétricas e térmicas de inversores para sistemas fotovoltaicos conectados à rede," Tese, 2010.

[11] D. W. Marquardt, "An algorithm for least-squares estimation of nonlinear parameters," Journal of the Society for Industrial and Applied Mathematics, vol. 11, no. 2, pp. 431-441, 1963. 